\title{
Naringenin and morin reduces insulin resistance and endometrial hyperplasia in the rat model of polycystic ovarian syndrome through enhancement of inflammation and autophagic apoptosis
}

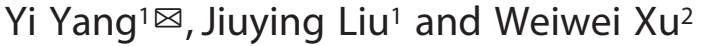 \\ 'Department of Gynecology and Obstetrics, Wuhan Children's Hospital (Wuhan Maternal and Child Healthcare Hospital), Tongji Medical College, \\ Huazhong University of Science and Technology, Wuhan, Hubei, 430030, P.R. China; ${ }^{2}$ Department of Gynecology and Obstetrics, Second Peo- \\ ple's Hospital of Huanggang, Huanggang, Hubei, 438315, P.R. China
}

Polycystic Ovary Syndrome (PCOS) is a gynecologic disorder with unsatisfactory treatment options. Hyperandrogenism and insulin resistance (IR) are two symptoms of PCOS. The majority of PCOS patients (approximately $50 \%$ to $70 \%$ ) have IR and moderate diffuse inflammation of varying degrees. We investigated in-vitro and invivo effects of naringenin, morin and their combination on PCOS induced endometrial hyperplasia by interfering with the mTORC1 and MTORC2 signaling pathways. The vaginal smear test ensured the regular oestrous cycles in female rats. Serum cytokines (TNF-a \& IL-6) were assessed using the ELISA test, followed by in-vivo and in-vitro determination of prominent gene expressions (mTORC1\& C2, p62, LC3-II, and Caspase-3 involved in the inflammatory signaling mechanisms through RTPCR, western bloting, or immunohistochemical analysis. In addition, the viability of naringenin or morin treated cells was determined using flow cytometry analysis. The abnormal oestrous cycle and vaginal keratosis indicated that PCOS was induced successfully. The recovery rate of the oestrous cycle with treatments was increased significantly $(P<0.01)$ when compared to the PCOS model. Narigenin, morin, or a combination of the two drugs substantially decreased serum insulin, TNF-a, IL- 6 levels with improved total anti-oxidant capacity and SOD levels $(P<0.01)$. Treatments showed suppression of HEC-1-A cells proliferation with increased apoptosis $(P<0.01)$ by the upregulation of Caspase- 3 expression, followed by downregulation of $\mathrm{mTORC}$, MTORC1, and p62 $(P<0.01)$ expressions with improved LC3-II expressions $(P<0.05)$ respectively. The histological findings showed a substantial increase in the thickness of granulose layers with improved corpora lutea and declined the number of cysts. Our findings noticed improved inflammatory and oxidative microenvironment of ovarian tissues in PCOS treated rats involving the autophagic and apoptotic mechanisms demonstrating synergistic in-vitro and invivo therapeutic effects of treatments on PCOS-induced endometrial hyperplasia.

Keywords: PCOS, Morin, Naringenin, hyperplasia

Received: 01 June, 2021; revised: 05 August, 2021; accepted: 27 October, 2021; available on-line: 10 February, 2022

凶e-mail: yiyang979@yahoo.com

Abbreviations: COX-2, Cyclooxygenase-2; DAB3, 3'-diaminobenzidine; FBG, Fasting blood glucose; HEC-1-A, Human endometrial adenocarcinoma cells; hCG, Human chorionic gonadotropin; IL-6, Interleukin-6; MAPK, mitogen-activated protein kinase; MTORC1, mammalian target of rapamycin complex 1; LC3-II, Microtubule- associated protein light chain 3-2; NO, Nitric oxide; PCOS, olycystic ovarian syndrome; SOD, Superoxide dismutase; TNF-a, Tissue necrosis factor

\section{INTRODUCTION}

Insulin resistance and hyperandrogenism are the main causes of polycystic ovary syndrome (PCOS), which affects about $15 \%$ of women of reproductive age (Moran et al., 2011). Premalignant lesions are called endometrial hyperplasia $(\mathrm{EH})$. It was estimated, however, that approximately $3-12 \%$ of endometrial hyperplasia cases lead to infiltrating cancer. The incidence of $\mathrm{EH}$ in women with PCOS without treatment of contraceptive steroids was over 30\% (Kurman et al., 1985). As per the previous study, 25\% and 2\% of Polycystic Ovarian Syndrome patients developed simple EH and EC respectively(Cheung, 2001). Unlike endometrial cancer (EC), simple endometrial hyperplasia was precluded as a pre-malignant condition and infrequently lead to endometrial cancer (Kurman et al., 1985). The levels of many endometrial markers experience major changes during the implantation of the embryos, suggesting that simple endometrial hyperplasia can be initiated before polycystic ovary syndrome induced infertility (Park et al., 2011). The EH decreased the rates of embryos implantation (Goncharenko et al., 2013; Ip et al., 2013). The simple endometrial hyperplasia treatment could enhance the pregnancy rate and decrease the abortion rate in PCOS patients. There was evidence that cardio-metabolic risk, insulin resistance, and weight gain are associated with PCOS (Ardawi \& Rouzi, 2005; Bulun et al., 2005; Rakha et al., 2012). Obesity also induced insulin resistance through the release of tumor necrosis factor (TNF)- $\alpha$ and PPAR-dependent pathways (Barber et al., 2016).

Phytoestrogens are present in different plant types and include flavonoids, coumestan (Neisy et al., 2019) are capable of binding to the oestrogen receptor (ER) (Miksicek, 1994; Ververidis et al., 2007). Phytoestrogens display several actions through reduced pro-inflammatory COX-2 and NO synthase (Kuiper et al., 1998); boosting the expression of GLUT-4 transporter triggering sensitivity of insulin; antioxidant genes activation and adipogenesis inhibition by interfering the AMPK signaling cascade (Medjakovic et al., 2010).

The beneficial actions of foods containing naringenin and morin on oestrogen deficiency diseases had been identified in the past. Breast cancer (linked to oestrogen deficiency) was found to be substantially less common in 
areas where flavonoid-rich foods are consumed (Harris et al., 2004; Palma-Duran et al., 2015).

According to the research, autophagy de-regulation is said to be an important part of the development of EH in PCOS patients (Sarkaki et al., 2015; Zhang et al., 2017), and both naringenin and morin have been shown to have a synergistic therapeutic effect on autophagy (Lin et al., 2018; Singh et al., 2019). The naringenin had effectively deactivated mTORC2 signaling in HCC-94 cells and further improved its effects on MAPK signaling and mTORC1 suppression (Raha et al., 2015; Zhang et al., 2018). However, the effects of morin on inhibition of the MAPK and mTORC1 and signaling pathways were not studied. The naringenin and morin were shown to improve the auto-phagocytosis and programmed cell death of HCC-94 cells (Lin et al., 2018; Raha et al., 2015; Singh et al., 2019; Zhang et al., 2018). Therefore, the pro-autophagy treatment offered by naringenin and morin can give an ideal route for treating HEC-1-A-cells. Hence, our study involved the investigation of in-vitro and in-vivo beneficial effects of naringenin, morin, and their combined treatment on PCOS induced endometrial hyperplasia through the inhibition of the proliferation of HEC-1-A-cells by interfering with the mTORC1 and mTORC2 signaling pathways.

\section{MATERIALS AND METHODS}

\section{Experimental design}

Female rats of two months old were used for the study. All rats (60) were received clean water in separate pathogen-free animal facilities and food was provided. The rats were maintained at a temperature of $30^{\circ} \mathrm{C}$. All rats were tested before the start of the analysis to ensure that they had regular oestrous cycles. A standard procedure for vaginal smear test confirmed the existence of regular oestrus cycles. The rats were subsequently assigned to control, polycystic ovary syndrome (PCOS), PCOS+naringenin, PCOS+morin, and $\mathrm{PCOS}+$ naringenin + morin groups. Naringenin (purity $>$ $8 \%, 67604-48-2$ ) and Morin (purity $>97 \%, 480-16-0$ ), both were procured from Merck, USA. A homogenous suspension of naringenin and morin was prepared separately using sodium carboxymethylcellulose (CMS, 1\% $\mathrm{w} / \mathrm{v}$ ) suitable for oral administration.) Each group was comprised of 12 rats. The control rats were given saline only. The insulin + hCG (Human chorionic gonadotropin) was given to rats in the PCOS group to stimulate both symptoms of excess androgen and higher insulin levels in the blood. The insulin+hCG treatment was given as per standard procedures and doses followed a method previously described ( $\mathrm{Li}$ et al., 2013; Tamadon et al., 2018). Insulin was first administered at a dosage of 0.5 IU per day, then steadily augmented to $5 \mathrm{IU} /$ day to induce hyperinsulinemia and insulin resistance. PCOS induction was deemed successful in rats with keratosis in their vaginal epithelial cells for 10 days (Yu et al., 2014). PCOS rats' orbital venous blood was obtained and tested after 15 hours for fasting insulin and blood glucose concentrations. The evaluation of the homeostatic model (Keskin et al., 2005) HOMA-IR=Fasting Blood Glucose $(\mathrm{mmol} / \mathrm{L})$ - Fasting Insulin concentration (mU/L) per 22.5 was evaluated in conjunction with the HOMA-IR. The Insulin Resistance in PCOS rats with HOMA-IR was greater than 3.0 (Coniglio et al., 2012). The hCG was administered to trigger hyperandrogenism with 3.0 $\mathrm{IU}$ per day after insulin resistance and hyperinsulinemia were confirmed. The subcutaneous injections of hCG twice a day were given to all rats until the experiment was completed.

In the PCOS+naringenin group, the rats received a dose of $200 \mathrm{mg}$ per $\mathrm{kg}$ of naringenin dissolved in sodium carboxymethylcellulose (CMS, 1 percent w/v) orally on the 23rd day of the test using gavage. Similarly, in the PCOS + Morin group, the rats received oral $200 \mathrm{mg}$ per $\mathrm{kg}$ of morin dissolved in sodium carboxymethylcellulose (CMS, 1 percent $\mathrm{w} / \mathrm{v})$. The PCOS+naringenin+morin group received orally $200 \mathrm{mg}$ per $\mathrm{kg}$ of naringenin dissolved in sodium carboxymethylcellulose (CMS, 1 percent $\mathrm{w} / \mathrm{v}$ ), plus $200 \mathrm{mg}$ per $\mathrm{kg}$ of morin dissolved in sodium carboxymethylcellulose (CMS, 1 percent w/v) respectively.

The blood was collected from each rat following anesthesia after the study. The uterus of the rats was also taken for a real-time polymerase reaction and a Western blot examination. Ovaries of all treated rats were collected surgically, set in formalin, and then placed in alkanes (paraffin). The $5 \mu \mathrm{m}$ thick sections were stained with eosin and hematoxylin until the morphological features of the ovaries were blindly evaluated by two pathologists. The research protocol was carried out with the permission of the Institute's Animal Ethics Committee under the national and international guidelines for the care and use of laboratory animals.

\section{Fasting Plasma Glucose}

The blood was collected after anesthesia following fasting for 15 hours from the orbital vein. The blood was measured for fasting plasma glucose with a glucometer.

\section{Insulin Release Test}

The insulin production was evaluated in rats that had been fasted for 15 hours. The rats were treated with glucose by gavage to the normal dosage of $4 \mathrm{~g} / \mathrm{kg}$ body weight. Blood was collected immediately after oral administration from the orbital vein at $30 \mathrm{~min}, 60 \mathrm{~min}$, and 120 min after gavage. For 20 minutes, the blood was centrifuged at $4500 \mathrm{rpm}$. The serum was then placed in a freezer at $-80^{\circ} \mathrm{C}$ for further studies.

At each stage, the blood insulin was measured using an immunosorbent assay kit connected to the insulin enzyme-linked. A microplate reader at $480 \mathrm{~nm}$ was used to measure the optical density values. The blood insulin release curve (IUC) area was calculated as follows: IAUC $=$ FINS $\times 0.25+60$-minute insulin $\times$ 0.75+120-minute insulin $\times 05$ (Bahceci et al., 2004).

\section{Enzyme-Linked Immunosorbent Assay (ELISA)}

Superoxide dismutase function, total antioxidant potential, and total oxidative stress levels in rat ovarian tissues were determined utilizing ELISA kits as per the manufacturer's instructions to ascertain the antioxidant effects of naringenin or morin or their combinations. The activity of SOD was expressed as mol per $\mu \mathrm{g}$ protein and total antioxidant potential and total oxidative stress levels were expressed as nmol per mg protein after determining the relative protein content in each sample.

\section{Culture of cells}

The Human endometrial cancer-1-A cells were collected and seeded on a 96-well plate 50 hours after surgery. The cells were cultivated in a Dulbecco modified Eagle medium (SigmaAldrich, USA) with 12 percent fe- 
tal calf serum, 80 and $120 \mu \mathrm{g}$ per $\mathrm{ml}$ of penicillin and streptomycin respectively at $40^{\circ} \mathrm{C}$ with the supply of 5 percent $\mathrm{CO}_{2}$. The cells were sub-cultured once or twice a week and harvested for subsequent experiments after a specified exponential growth period. The HEC-1-A cells were divided into four classes to understand the effect of morin and naringenin on multiple factors associated with cell proliferation and apoptosis and treated accordingly: (a) control group; (b) naringenin group $(500 \mu \mathrm{M}$ of naringenin); (c) Morin group (500 $\mu \mathrm{M}$ of morin), and (d) combined treatment group $(500 \mu \mathrm{M}$ of naringenin and $500 \mu \mathrm{M}$ of morin) to investigate the outcome of morin and naringenin on cellular proliferation and cell deathrelated factors. Cells were extracted from treated and untreated groups after 2 days of therapy to test the levels of the corresponding cellular markers. The experiments were replicated three times.

\section{Cell proliferation assay}

The Human endometrial cancer-1-A cells were collected and seeded on a 96-well plate 50 hours after surgery (In $100 \mu \mathrm{l}$ of cell culture medium, with a density of $10^{5}$ cells per well). To 90 percent confluence of the cells, various naringenin or morin or combined concentrations at $0 \mu \mathrm{mol} / \mathrm{L}, 10 \mu \mathrm{mol} / \mathrm{L}, 20 \mu \mathrm{mol} / \mathrm{L}, 40 \mu \mathrm{mol} / \mathrm{L}$, $60 \mu \mathrm{mol} / \mathrm{L}$, and $80 \mu \mathrm{mol} / \mathrm{L}$ were applied to the cell culture medium. The cells were then incubated for 4.5 hours in the dark with a reagent after 48 hours of incubation. To evaluate the viability of the cells, their optical density was measured at $480 \mathrm{~nm}$. There were three repetitions of each experiment.

\section{Immunohistochemistry}

The levels of inflammatory cytokines such as IL-6and TNF- $\alpha$ in the rat ovaries were determined using immunohistochemistry on tissue slices. The samples were fixed in 3 percent formaldehyde, engrained in paraffin, and sectioned into $5 \mu \mathrm{m}$ thick parts for the assay. The sections were again incubated for one hour at $70^{\circ} \mathrm{C}$ before being dewaxed in xylene and dehydrated in alcohol. Following that, the sections were incubated for $45 \mathrm{~min}-$ utes at room temperature in 2 percent hydrogen peroxide to inhibit endogenous peroxidase activity. The samples were then blocked with 4 percent goat serum for 45 minutes at room temperature before being incubated with primary antibodies (1:2000 dilutions) overnight at $4^{\circ} \mathrm{C}$. The sections were incubated with the secondary antibodies of goat-antirabbit conjugated with biotin (1:2000 dilutions) for one hour at room temperature on the next day. Additionally, the sections were stained for $15 \mathrm{~min}$ utes with $\mathrm{DAB}$, then counterstained with hematoxylin for 5 minutes at room temperature, and briefly dehydrated in ethanol, and mounted in neutral gum for examination under the microscope.

\section{Western blot analysis}

The supernatant was obtained after the cells specimens were lysed in a lysis buffer and separated by centrifugation at $14000 \mathrm{rpm}$ for 15 minutes. A bicinchoninic acid assay kit was used to assess the protein content in the lysate. Following that, isolated protein samples (30 $\mu \mathrm{g}$ each) were inactivated by boiling for 10 minutes at $80^{\circ} \mathrm{C}$ before being electrophorsed on a $10 \%$ SDS. The protein components were then transferred onto a nitrocellulose membrane followed by blocking for 2 hours with 3\% skim milk, then incubated overnight with primary antibodies against mTORC1 (catalogue number: ab120224; 1:2000; Abcam, USA) \& mTORC2 (catalogue number sc-1549; 1:2000; Santa Cruz Biotechnology), p62 (catalogue number - ab91526; 1:2000; Abcam, USA), Caspase-3 (catalogue number - ab145046;1:2000; Abcam, USA), LC3-II (catalogue - number-ab192890; 1:2000; Abcam, USA) and GAPDH (catalogue number - ab8245; 1:4000; Abcam, USA). The membrane was incubated with secondary antibodies for one hour at room temperature on the second day (1:2000; Abcam, USA). The membrane was fully dipped in a chemiluminescence reagent after three additional PBS washes, and the gel colour produced in the dark was visualized. The target and the GAPDH protein bands ratio were used to measure the mTORC1 and mTORC2, LC3-II, p62, and Caspase-3 relative protein expressions. Each experiment was carried out three times in total.

\section{Real time PCR assessment}

The HEC-1-A cells $(1106 / \mathrm{ml})$ were cultivated in six well plates. The cells were harvested 4 hours after treatment with naringinein, morin, or their combination, and were used to conduct the experiments. TRIzol reagent (Thermofisher Scientific, CA, USA) was used to extract total RNA, which was then reversed transcribed into cDNA using RNA assay kit. The cDNA samples were combined with SYBR-Green PCR-Mastermix (Sigmaldrich, USA) and TNF- $\alpha$, IL- 6 , and $\beta$-actin primers. A Bio-rad thermal Cycler was used to perform quantitative real-time PCR assessment (Liu et al., 2016).

\section{Apoptosis analysis}

The HEC-1-A cells were washed with PBS three times after 2 days of treatments and flow cytometry was used to detect cell apoptotic profiles with annexin V-fluorescein detection kit following the manufacturer's instructions.

\section{Statistical analysis}

Using SPSS 21.0 software, variance analysis was carried out. The mean \pm SEM was expressed for all datasets. All experiments were replicated three times. The student's $t$-test was applied to compare the inter-group statistical significance. For time-course studies, two-way ANOVA was used to analyze the data. If the 2-way ANOVA showed significant variation then Bonferroni's post-hoc test was applied to assess the intergroup variation. The $P<0.05$ was considered statistically significant.

\section{RESULTS}

\section{Insulin tolerance in a rat model of PCOS}

The rats' vaginal cytological changes were examined using the methylene blue staining technique after 24 days of hCG injections. There was an abnormal oestrous cycle and vaginal keratosis in a group of 75 rats, 48 indicated that PCOS was successfully induced following the assessment of FBS and fasting insulin. All the PCOS rats showed Homeostatic Model Assessment of Insulin resistance $>2.91$. In PCOS rats, the incidence rate of IR was 66.1 percent

\section{Oestrous cycles improved, weight increased and ovary co-efficient increased with treatments}

The rats in the naringenin, morin, and naringenin + morin groups showed restored oestrous cy- 
A)

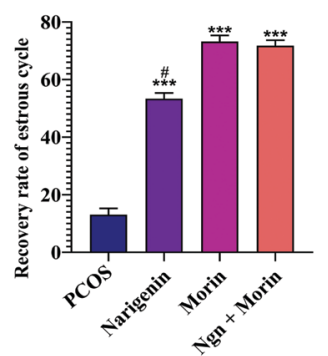

C)

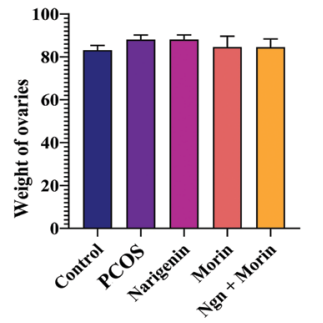

B)

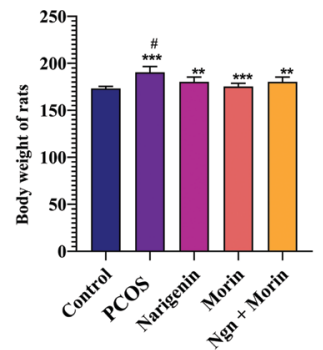

D)

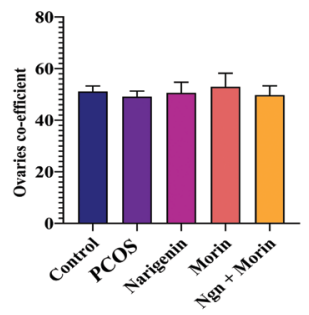

Figure 1. The effects of naringenin (Ngn), morin and their combination on polycystic ovary syndrome (PCOS) rats.

A) Recovery rate of oestrous cycles. B) Rat body weights. C) Weight of ovaries. D) ovary coefficient. Data was statistically analysed using Student's $t$-test. ${ }^{*} P<0.05 v s$ PCOS group; ${ }^{*} P<0.01$ and ${ }^{* * *} P<0.001$ vs control \&PCOS group respectively. $\mathrm{N}=12$ rats.

cles after treatment. The recovery rates were 8.33 percent, 58.33 percent, 66.67 percent, and 76.54 percent respectively in the model, naringenin, morin, naringenin + morin groups (Fig. 1A). After treatment, the naringenin, morin, and the combined groups had lower body weights (Fig. 1B). The ovarian ratio was evaluated as follows: ovarian coefficient $=$ ovarian weight per body weight $(\mathrm{mg} \times$ 100 per gram) (Coniglio et al., 2012). Ovarian weight and ovarian coefficients did not vary statistically significant from the model coefficients in the naringenin, morin, and naringenin + morin groups (Fig. 1C and D).

\section{Rat Ovarian Morphology}

The therapeutic effect of naringenin or morin or both on rat ovaries in PCOS was linked to the morphology of rat ovaries in various groups. The control groups'
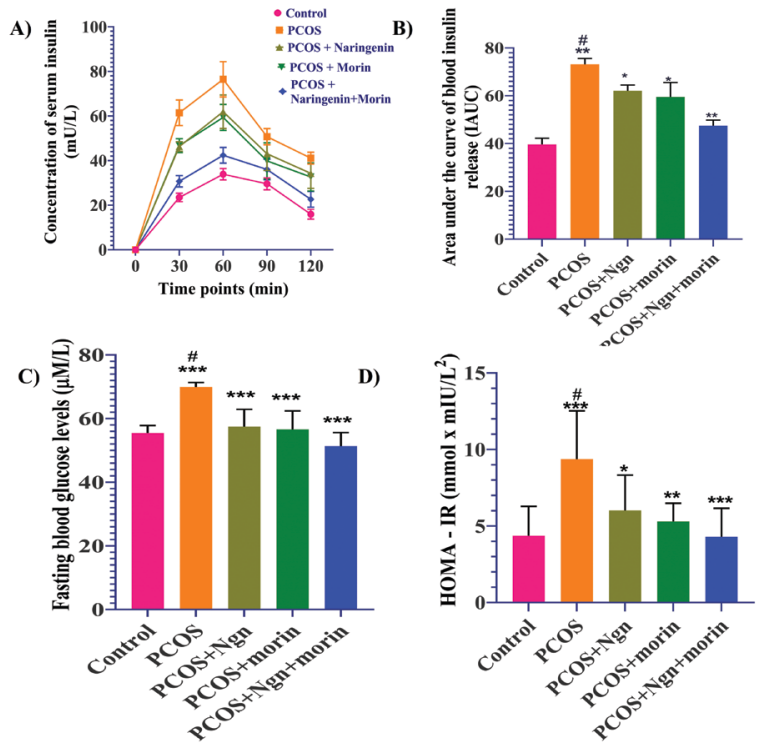

Figure 3. The effects of naringenin (Ngn), morin and their combination on serum insulin levels in PCOS rats

A) Serum insulin levels in rats. The release of insulin in rats at 30 min $(P<0.01$; Naringenin or morin or Naringenin+morin groups vs PCOS group); 60 min $(P<0.01$; Naringenin or morin or Naringenin +morin groups vs $P C O S$ group); $90 \mathrm{~min}(P<0.05 ; P<0.01$; Naringenin or morin or Naringenin+morin groups vs PCOS group); 120 min $(P<0.01 ; P<0.01$; Naringenin or morin or Naringenin + morin groups vs PCOS group). B) Area under the curve of blood insulin release (IAUC). C) Fasting blood glucose levels. D) Homeostasis model of assessment of insulin resistance (HOMA-IR). Data was statistically analysed using Student's $t$-test. Data from time course studies was analysed using two-way ANOVA and Bonferroni's post-hoc test. ${ }^{*} P<0.05$ vs PCOS group; ${ }^{* *} P<0.01$ and ${ }^{* * *} P<0.001$ vs control \&mod4el group respectively. $\mathrm{N}=12$ rats.

ovaries had multiple lutea follicles at different locations, with up to nine granulosa layers in the prevailing follicle. The PCOS group ovaries showed cystic dilatation without oocyte and corona radiation of the follicles. There was a decrease in the number of granulosa cells within the follicle by 3 layers and a decrease in the number of luteal cells. At different points, the naringenin, morin, or combined group ovaries showed follicles and more lutea
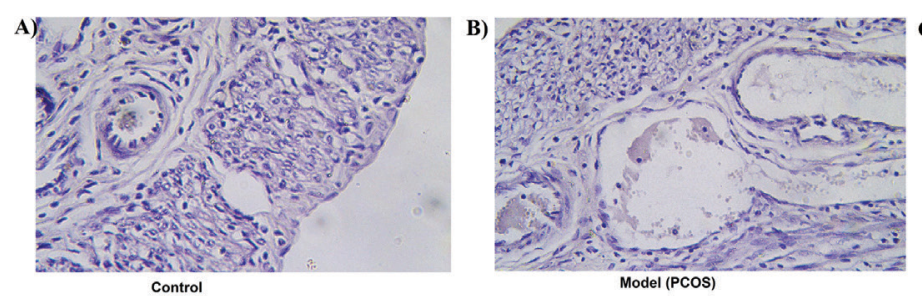

E)

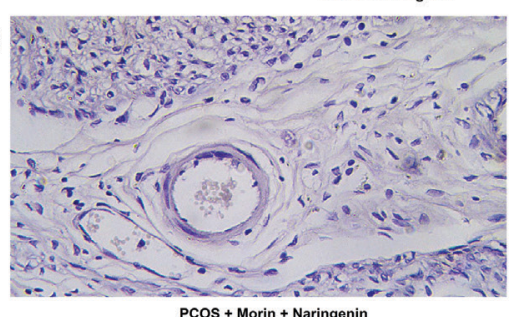

D)

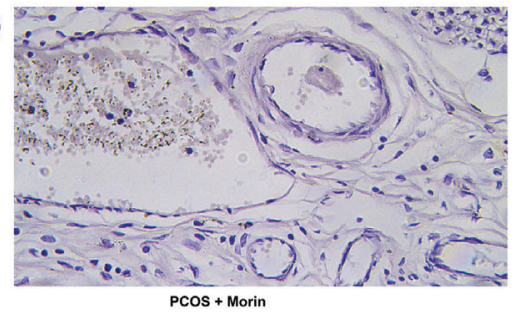

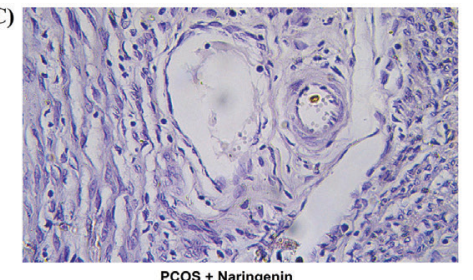

cos + Morin + Naringenin

Figure 2. The effects of naringenin (Ngn), morin and their combination showed improved the morphology of the ovarian follicles and increased the granulosa cells of the PCOS rats.

A) Control, B) PCOS model C) PCOS model + naringenin treatment, D) PCOS model +morin treatment and E) PCOS model+combined treatment (naringenin+morin). (Magnification $\times 100$ ). 

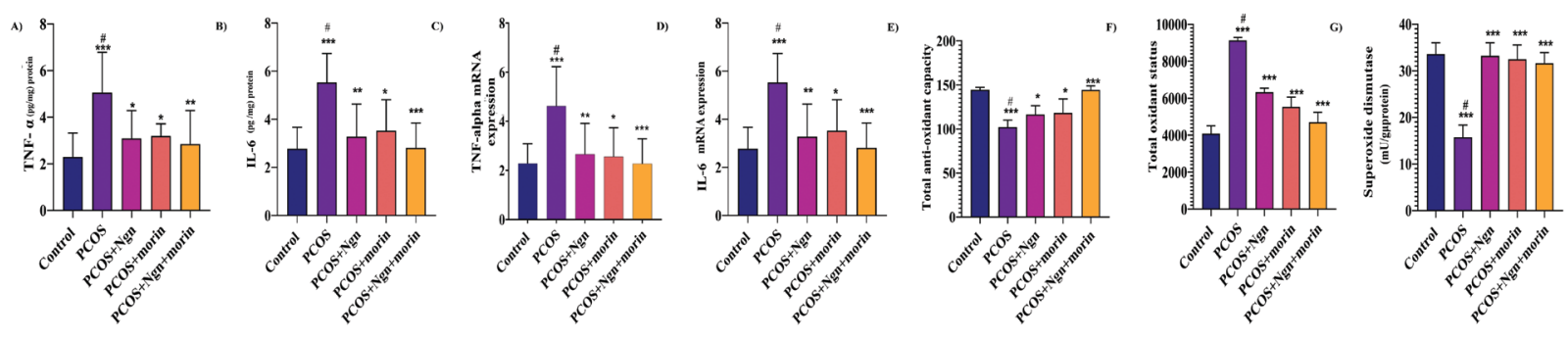

Figure 4. The effect of Naringenin (Ngn), morin and their combination on the concentration of (A) tumour necrosis factor- $a$ (TNF- $a$ ) (pg/mg protein), (B) interleukin-6 (IL-6) (pg/mg protein), (C, D) relative expression of TNF-a and IL-6 mRNA in the rat ovary, (E) the total antioxidant capacity (TAC) (F) the total oxidant status (TOS) and (G) the activity of the superoxide dismutase (SOD) in the ovary tissue of the PCOS control group compared to the treated groups and control group.

Data are represented as mean \pm S.E.M. ( $\mathrm{n}=12$ in each group). Data was statistically analysed using Student's $t$-test. *Significantly different between treated groups and PCOS control group. Significantly different from the control group $\left({ }^{*} P<0.05,{ }^{* *} P<0.01,{ }^{* * *} P<0.001\right)$

with the granulosa cells increasing in layers (3-7) compared to PCOS rats (Fig. 2).

\section{Release of serum insulin assessment}

An insulin release was established at various time points. The PCOS group had higher insulin levels at 30 min, $60 \mathrm{~min}$, and 120 minutes than the control group. The levels of insulin, as well as IAUC and FBG at different times, were all lower than in the PCOS group as compared to naringenin, morin, and combined groups (Fig. 3A-C; $P<0.05$ ). The Homeostatic model assessment-IR value was significantly greater than the control value $(P<0.01)$ in the PCOS group. The HOMA-IR values of naringenin, morin, and the combined groups, on the other hand, were considerably lesser when compared to the PCOS group (Fig. 3D; $P<0.01$ ).

\section{Interleukin-6 and TNF-a protein levels}

To observe the therapeutic effects of naringenin, morin, and their combination on inflammatory cytokines, immunohistochemistry was performed on tissues to compare Interleukin (IL)- 6 and TNF- $\alpha$ levels among the different groups. Compared to the control group, the levels of Interleukin-6, TNF- $\alpha$ were high in the PCOS group. Naringenin, morin, and combined therapy have substantially inhibited the levels of these inflammatory cytokines (Fig. 4). Moreover, naringenin,morin and their combination significantly suppressed the mRNA expression of TNF- $\alpha$ and IL-6 in PCOS group as compared to control rat (Liu et al., 2016). In the results of the PCOS group, the accumulation of oxidants was substantially greater with a significant decrease in the total antioxidant capacity $(P<0.05)$ than in the control group (Fig. 4). When compared to the PCOS group, the total antioxidant potential in the naringenin or morin or their combined groups was significantly higher $(P<0.01)$. In contrast, when compared to the PCOS group, different doses of naringenin or morin, or their combinations, drastically $(P<0.001)$ decreased total oxidant stress in ovarian tissues of treated groups. Additionally, the activity of the SOD in the ovarian tissue of the naringenin or morin or their combined groups was considerably higher $(P<0.001)$ than in the control.

\section{Effects of naringenin and morin on endometrial and myometrial regions}

The endometrial (Fig. 5a), myometrial (Fig. 5b) areas of the PCOS group were elevated relative to those

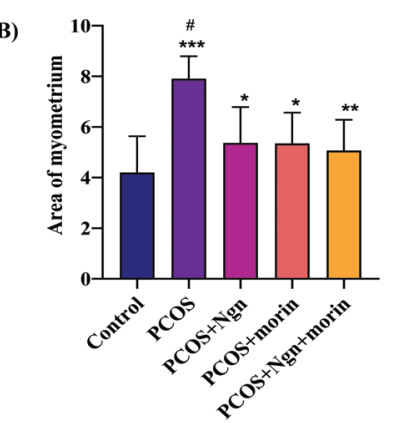

Figure 5. Effect of naringenin (Ngn), morin and their combination on the areas of endometrium and myometrium.

The naringenin and morin significantly reduced the areas of the endometrium (A) and myometrium (B) in PCOS, and the inhibitory effect of naringenin+morin was highly significant when compared to the control and PCOS groups. Data was statistically analysed using Student's $t$-test. ${ }^{*} P<0.05$, ${ }^{* *} P<0.01,{ }^{* * *} P<0.001$

of the control group, as shown in Fig. 5. Although the endometrial and myometrial areas of naringenin, morin, and the combined groups were all decreased, the greatest inhibitory effect on endometrial areas was shown that in PCOS rats than on myometrium. The combined therapy was found to be more effective than naringenin or morin alone (Fig. 5).

\section{Impact on mTORC-1, mTORC-2, LC3-II, p62 protein, and} Caspase-3 levels of naringenin and morin in-vivo

To learn more about the mechanisms that underpin the role of naringenin and morin in the polycystic ovary syndrome, the mTORC-1, mTORC-2, Light Chain 3-II, and Caspase- 3 levels were compared between the groups. The mTORC1 protein level (Fig. 6a) was much higher in the PCOS group than in the control group, as indicated in Fig. 6, whereas the abnormal mTORC1 protein levels were restored by treatment with naringenin or morin or their concomitant treatment. The abnormal levels of mTORC-1 in the PCOS+naringenin group were restored to a level similar to that of the PCOS+morin group. Similarly, the abnormal mTORC2 protein levels were restored after treatment with naringenin or morin or their combined treatment.

The PCOS group had slightly lower LC3-II (Fig. 6b) and Caspase-3 (Fig. 6c) protein levels than the control group, but naringenin or morin therapy partially restored 

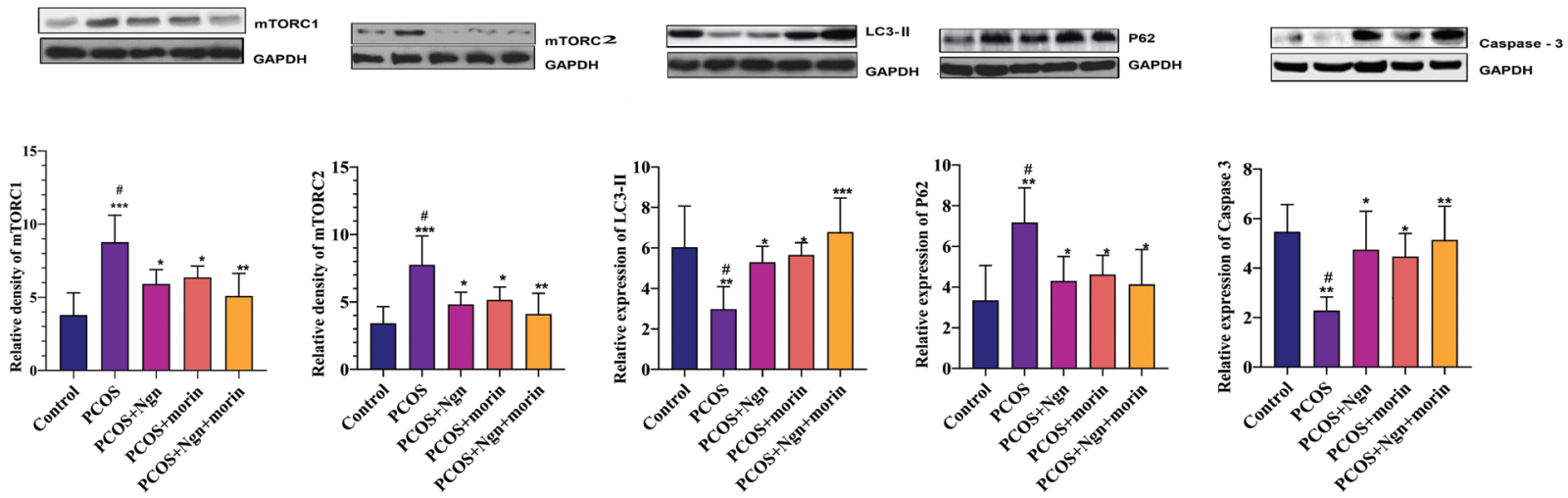

Figure 6. Effect of naringenin (Ngn), morin and their combination on the protein expression of mTORC1, $m$ TORC2 the levels of LC3-II, p62 AND Caspase-3 were detected by western blot analysis.

Data was statistically analysed using Student's $t$-test. *indicates significantly different between treated groups And PCOS control group $\left({ }^{*} P<0.05,{ }^{* *} P<0.01,{ }^{* * *} P<0.001\right)$ respectively.

the reduced LC3-II and Caspase-3 expressions in PCOS rats. However, there was a significant impact noticed with the combined treatment (naringenin+morin)in the LC3-II and Caspase protein levels. The up-regulated p62 (sequestosome $1 / \mathrm{p} 62$ is a multifunctional protein and a signaling hub that shuttles ubiquitinated proteins to the lysosome during autophagy) expressions in PCOS rats were inhibited by naringenin or morin therapy compared to the control group, and the greatest inhibitory effect on p62 expressions was noticed with the combined treatment of naringenin and morin therapy, which displayed a degree of p62 expression close to that in the control group.

\section{The effects of naringenin and morin therapy on the proliferation of cells and apoptosis}

A flow cytometry analysis was used to evaluate the apoptotic and viability of $\mathrm{HEC}-1-\mathrm{A}$ cells treated with naringenin, morin, or naringenin+morin. The HEC-1-A cell viability was slightly lower in the naringenin and morin group than in the PCOS group, while cell viability for the combined treatment (naringenin+morin) was the lowest (Fig. 7a). The naringenin or morin or their combined treatment also substantially improved the ability to cell apoptosis of both treated and untreated apoptotic HEC-1-A cells (Fig. 7b). There was a comparable impact of naringenin and morin on cell apoptosis and the combined effect (naringenin + morin therapy) showed

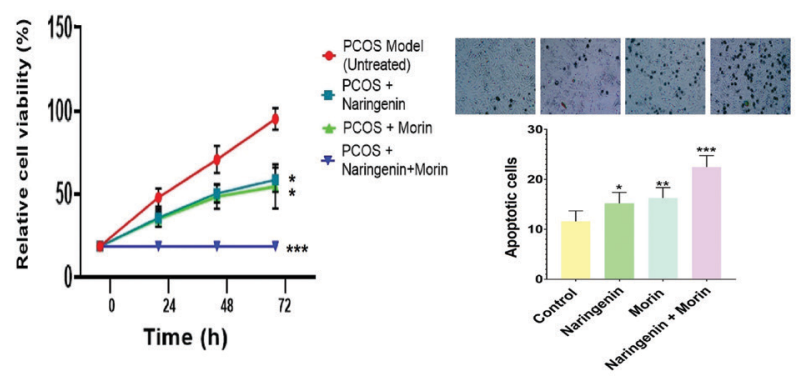

Figure 7. Proliferation and apoptosis of HEC-A-1 cells were affected by the treatment with naringenin (Ngn), morin and their combination.

Data was statistically analysed using Student's $t$-test. Data from time course studies was analysed using two-way ANOVA and Bonferroni's post-hoc test. *Indicates significantly different between treated groups and PCOS control group $\left({ }^{*} P<0.05,{ }^{*} P<0.01\right.$, $\left.{ }^{* * *} P<0.001\right)$ respectively. a synergistic effect. The active caspase-3 levels of the HEC-1-A cells treated with naringenin and morin were significantly higher than the control group. The combined treatment showed the highest caspase-3 levels compared to the control group. Naringenin and morin reduced mTORC1 and mTORC2 expressions that triggered autophagy by downregulation causing apoptosis of cells, which increased Caspase-3 and LC3-II levels. The combined treatment of naringenin and morin can be used as a unique strategy in treating $\mathrm{EH}$ induced by polycystic ovary syndrome since cell apoptosis showed a significant role in $\mathrm{EH}$ production during polycystic ovary syndrome.

\section{DISCUSSION}

Asians have a lower incidence of oestrogen-related disease than Westerners, which has prompted researchers to investigate the therapeutic agents in relevant plants. The therapeutic effects of naringenin and morin in oestrogen related disorders such as PCOS were scarce (Rehman et al., 2018; Roy et al., 2016). The most significant characteristic of naringenin or morin was its estradiollike structure and phytoestrogen production (Rehman et al., 2018; Roy et al., 2016). The previous research had focused primarily on the anti-inflammatory and antioxidant actions of naringenin in PCOS. The anti-inflammatory and antioxidant effects may be linked to KEAP up-regulation and iNOS and COX-2 down-regulation, which are part of the effects of phytoestrogens (Kandemir et al., 2020). Keap1 (Keech-like ECH-associated protein 1) is required for host defense towards oxidative and electrophilic damage. Keap1 acts as an adapter for ubiquitin E3 ligase and facilitates proteasomal degradation in the absence of stress (Taguchi et al., 2012). Several studies were shown that naringenin or morin can inhibit the cascade of the NF-kB signal inflammation and decrease the levels of inflammatory cytokines (including tumor necrosis factor- $\alpha$ and Interleukins) (Arisha et al., 2019; Wali et al., 2020). Previous diabetes and its complications research have shown that by treating both hyperglycemia and oxidative stress using animal models, naringenin or morin had a protective role as an antidiabetic agent (Arisha et al., 2019; Wali et al., 2020).

In the current analysis, we successfully developed a rat PCOS model. Typically, the metabolic and reproductive characteristics resembling to human PCOS, such as 
irregular reproductive cycles, increase in weight of the body, ovarian co-efficient, and elevated FBG and exogenous insulin levels were reproduced in the rat model. The injected rats showed insulin resistance in 66.7 percent of cases. In rats with PCOS, the oestrous cycle was restored by treatment with morin or naringenin. The body weights of the PCOS rats, ovarian morphology, and serum insulin levels were increased. These findings were shown that naringenin or morin induces a strong therapeutic effect on insulin-resistant PCOS in rats.

The etiology and pathology of PCOS were further discussed by recent studies, wherein long-term inflammation (besides IR and hyperandrogenism), especially low and persistent inflammation, is a hallmark of polycystic ovary syndrome (Escobar-Morreale et al., 2005). The immune response was associated with moderate chronic inflammation but without the presence of acute inflammatory symptoms including swelling, redness, pain, or heat (Lee \& Lee, 2014). Inflammation and IR's cause and effect were not well understood, IR and the coexistence of inflammation were widely recognized (EscobarMorreale et al., 2005; Lee \& Lee, 2014). This result indicates that oxidative stress has been correlated with IR in PCOS. The study showed that the PCOS group's IL-1 $\beta$, IL-6, and TNF-alpha expression levels were higher than those of the controls. Insulin is both a reproductive and a metabolic hormone, as evidenced by the link between the insulin resistance and PCOS. It modulates ovarian steroidogenesis by acting as a co-gonadotropin via its associated receptor. This effect is maintained despite resistance to insulin's metabolic activities in the peripheral and ovary. Ovulation appears to be dependent on insulin signalling in the central nervous system (CNS). Hyperinsulinemia boosts androgen production in PCOS, according to human research. Because reducing insulin levels has no effect on circulating androgen concentrations in healthy women, it appears that intrinsic defects in steroidogenesis are required for this insulin activity to occur. Insulin has an essential role in the synthesis of SHBG. Insulin resistance can be reduced, and ovulatory menstrual periods can be restored (Dunaif, 1997).

The morin effectively deactivated naringenin-induced mTORC2 signal activation in HEC-1-A cells as a result, combined treatment with morin and naringenin decreased HEC-1-A cell proliferation. The concomitant naringenin and morin therapy further decreases the invitro and in-vivo tumor cell proliferation by interrupting the mTORC1/mTORC2 signal response circuit. (Kumar et al., 2017; Rajput et al., 2021). The synergistic effect of naringenin and morin leads to the promotion of autophagy and apoptosis of HEC-1-A cells. An antiautophagic strategy could also be employed when naringenin and morin were used together to treat HEC-1-A. In the present research, the effect of naringenin and morin in the levels of mTORC-1, mTORC-2, LC3-II, and Caspase- 3 were investigated in western blot and immunohistochemistry studies. The results indicated that polycystic ovary syndrome decreased the concentrations of, LC3-II, and Caspase-3, while the treatment with naringenin increased them. In contrast, naringenin reduced the mTORC1, mTORC2, p62 levels regulated by PCOS. The effect of morin increased the levels of Caspase-3 and LC3-II with a reduction in the p62, mTORC1, and mTORC2 levels respectively.

A process called autophagy involves autophagosomes ingesting organelles and proteins from the cytoplasm. LC3-phosphatidylethanolamine conjugate (LC3-II) is formed when a cytosolic form of LC3 (LC3-I) is coupled to phosphatidylethanolamine and driven to autophago- somal membranes. During autophagosome fusion with lysosome formation, intra-autophagosomal elements are destroyed by lysosomal hydrolases, which are found in the lysosomes. Simultaneously, LC3-II is destroyed in the autolysosomal lumen. The autophagosomal marker LC3-II undergoes rapid turnover in response to starvation, and the detection of LC3 by immunofluorescence or immunoblotting has proven to be a consistent method for assessing autophagy-related mechanisms, such as autophagic cell death, in animal models of starvation (Tanida et al., 2008).

In response to cytotoxic stress, autophagy and apoptosis govern cancer cell survival; nevertheless, their causal link is unknown. p62/sequestosome 1 is a protein complex that also serves as a signalling hub during autophagy, transporting misfolded proteins components to the lysosome for degradation. Inhibiting autophagy increases p62 expression, and prior studies imply that p62 may induce apoptosis. The study findings showed that the protein p62 can modulate a caspase-8-dependent apoptosis against the $\mathrm{BH} 3$ mimic ABT-263 in a cell line model. It was demonstrated that up-regulation of p62 enhanced ABT-263-induced caspase-8 stimulation, which was Bax-dependent and occurred via mitochondrial amplification. Caspases- 8 dependence was demonstrated utilising caspase-8-deficient cells and caspase-8 siRNA. Ectopic wild-type p62 was found to co-localize with caspase- 8 and accelerate its self-aggregation in ABT263-treated cells, but not in p62 mutants with reduced ability to trigger apoptosis, as demonstrated by a bimolecular fluorescence test. In the presence of ABT-263 and an autophagy inhibitor, endogenous p62 was found to be co-localized with caspase- 8 . The autophagosome marker LC3II interacts and co-localizes with caspase-8, according to a study. The p62 gene was knocked out, which reduced the binding of caspase- 8 to LC3II, whereas the p62 gene was overexpressed, which increased the co-localization of caspase-8 aggregation with LC3. In contrast to the connection between caspase- 8 and p62, the LC3 knockdown had no effect on this interaction, suggesting that p62 may assist caspase- 8 translocation to the autophagosomal membrane. When TRAIL, a direct activator of caspase- 8 , was used alone or in combination with ABT-263, it caused caspase- 8 aggregation and colocalization with $\mathrm{p} 62$, which was associated with a synergistic pharmacological interaction, as reported previously. these findings suggest that up-regulation of p62 can cause apoptosis by activating caspase- 8 in the presence of autophagy inhibitory factors. LC3, the mammalian homolog of the yeast Atg8 protein, undergoes conversion from LC3-I to LC3-II during autophagy and acts as a valid marker of autophagosomes during lysosome degradation. Caspase-8-dependent and mitochondria-mediated apoptotic pathways can be enhanced by autophagy inhibition through unknown mechanisms (Huang et al., 2013)

Naringenin, a traditional hypoglycemic agent used to treat type 2 diabetes mellitus, may enhance hyperglycemic symptoms without causing endogenous secretion of insulin (Sarkar et al., 2012; You et al., 2016) The effect of naringenin on ischemic brain injury, Huntington disease, and Parkinson's disease, among other central nervous system disorders (Chtourou et al., 2015; Sarkar et al., 2012; You et al., 2016) were shown to be therapeutic that could minimize the intensity of $\mathrm{EH}$ caused by tamoxifen and estrogen, by inhibiting the activation of S6K1 signaling by mTOR (Dirimanov \& Högger, 2019). The study (Xu et al., 2011) also showed that naringenin can increase progesterone receptor expression by suppressing mTOR 
activation in EC cells. Naringenin has been shown to control cell differentiation and proliferation by acting as an anti-mitogen. It has been shown that mTOR signaling suppression effectively postpones the development of endometrial, breast, and ovarian cancers (MartínezRodríguez et al., 2020; Nowak et al., 2020; Treeck et al., 2006). Morin also improved ACR-induced hepatotoxicity and nephrotoxicity by modulating the protein levels of mTOR signalling pathways (Rajput et al., 2021).

The remarkable suppression of adhesion and migration of SKOV-3 and A2780 cell lines together with the distinct regulation of autophagy and significant apoptotic induction was noticed after treatment with morin, although the exact autophagic mechanism of morin is still unknown (Nowak et al., 2020; Pal Singh et al., 2020). Autophagy activation has been implicated in cisplatin resistance, while autophagy suppression improves the effectiveness of morin in SKOV-3 cells (Bieg et al., 2018; Pal Singh et al., 2020). A study found that morin can induce melanoma cell apoptosis with ROS regulating pro and anti-apoptotic proteins (Lee et al., 2019).

The flow cytometry and western blot analyses were used to evaluate the apoptotic and viability profiles of HEC-1-A cells treated with naringenin, morin, and their combination therapy. The findings discovered that naringenin and morin therapies inhibited HEC-1-A cell proliferation while increasing Caspase-3 expression, which facilitated apoptosis. The mTOR pathway can efficiently control autophagy action by activating chemotherapeutic effects and apoptotic mechanisms (Jung et al., 2010; Nicoletti et al., 2011). Autophagy can either facilitate or impede tumor cell formation, depending on the circumstances. In HEC-1-A cells, concomitant treatment of naringenin and morin will increase the level of autophagy. As a result, autophagy caused by naringenin and morin is anti-apoptotic rather than apoptotic. The mTOR can keep a balance of growth factors, uptake of glucose, amino acids, environmental stress, and hormones as a key change in many biological processes. The mTOR kinases were also strongly preserved in many organisms and participate in the regulation of stress autophagy. The two different signaling pathways, mTORC1 and mTORC2, mTOR kinases serve as catalytic components. The mTORC1 can stimulate p70S6K, which phosphorylates the ribosomal proteins (4E-BP1) and regulates protein translation. The mTORC2 complex, which consists of mTORRictor, mSIN1, and Protor-1 in reverse, directly stimulates Akt signaling by phosphorylating Serine 473. The results showed that naringenin or morin or their combined treatments while inhibiting p62 expression, increased mTORC1, mTORC2, and LC3-II expression (Kumar et al., 2017; Shahid Ali Rajput et al., 2021). It can be inferred that naringenin and morin can reduce the insulin resistance and endometrial hyperplasia in the rat model of PCOS via regulation of inflammation and autophagic apoptosis. Earlier studies also showed attenuating effects of naringenin and morin on mTOR signaling and caspse- 3 activity in different animal models and in vitro studies (Kucukler, 2020; Park, 2008). Furthermore, parallel to the findings of previous studies, naringenin and morin reduced expression of inflammatory cytokines and enhanced the level of endogenous antioxidants that might have decreased insulin resistance, improved glucose levels, and attenuated endometrial hyperplasia in PCOS model (Moran et al., 2011; Neisy et al., 2019).

\section{CONCLUSION}

Our findings demonstrated that the concomitant naringenin and morin therapy decreases the in-vitro and in-vivo tumor cell proliferation by interrupting the mTORC1/ mTORC2 signal response circuit. The synergistic effect of naringenin and morin leads to the promotion of autophagy and apoptosis of HEC-1-A cells. In the present research, the effect of naringenin and morin in the levels of mTORC-1, mTORC-2, LC3-II, and Caspase-3 were investigated. The results indicated that polycystic ovary syndrome decreased the concentrations of, LC3-II, and Caspase-3, while the treatment with naringenin increased them. In contrast, naringenin reduced the mTORC1, mTORC2, p62 levels regulated by PCOS. The effect of morin increased the levels of Caspase-3 and LC3-II with a reduction in the p62, mTORC1, and mTORC2 levels respectively. Consequently, we found the in-vitro and in-vivo synergistic therapeutic effects of naringenin and morin on PCOS-induced endometrial hyperplasia thus regulating the autophagy process.

\section{REFERENCES}

Ardawi MS, Rouzi AA (2005) Plasma adiponectin and insulin resistance in women with polycystic ovary syndrome. Fertil Steril 83: 1708-1716. https://doi.org/10.1016/j.fertnstert.2004.11.077

Arisha AH, Ahmed, MM, Kamel MA, AttiaYA, Hussein MMA (2019) Morin ameliorates the testicular apoptosis, oxidative stress, and impact on blood-testis barrier induced by photo-extracellularly synthesized silver nanoparticles. Environ Sci Pollut Res Int 26: 28749-28762. https://doi.org/10.1007/s11356-019-06066-1

Bahceci M, Tuzcu A, Canoruc N, Tuzun Y, Kidir V, Aslan C (2004) Serum C-reactive protein (CRP) levels and insulin resistance in nonobese women with polycystic ovarian syndrome, and effect of bicalutamide on hirsutism, CRP levels and insulin resistance. Horm Res 62: 283-287. https://doi.org/10.1159/000081973

Barber TM, Dimitriadis GK, Andreou A, \& Franks S (2016) Polycystic ovary syndrome: insight into pathogenesis and a common association with insulin resistance. Clin Med (Lond) 16: 262-266. https:// doi.org/10.7861/clinmedicine.16-3-262

Bieg D, Sypniewski D, Nowak E, Bednarek I (2018) Morin decreases galectin-3 expression and sensitizes ovarian cancer cells to cisplatin. Arch Gynecol Obstet 298: 1181-1194. https://doi.org/10.1007/ s00404-018-4912-4

Bulun SE, Imir G, Utsunomiya H, Thung S, Gurates B, Tamura M, Lin Z (2005) Aromatase in endometriosis and uterine leiomyomata. I Steroid Biochem Mol Biol 95: 57-62. https://doi.org/10.1016/j. jsbmb.2005.04.012

Cheung AP (2001) Ultrasound and menstrual history in predicting endometrial hyperplasia in polycystic ovary syndrome. Obstet Gynecol 98: 325-331. https://doi.org/10.1016/s0029-7844(01)01432-6

Chtourou Y, Slima AB, Gdoura R, Fetoui H (2015) Naringenin mitigates iron-induced anxiety-like behavioral impairment, mitochondrial dysfunctions, ectonucleotidases and acetylcholinesterase alteration activities in rat hippocampus. Neurochem Res 40: 1563-1575. https:// doi.org/10.1007/s11064-015-1627-9

Coniglio RI, Meroño T, Montiel H, Malaspina MM, Salgueiro AM, Otero JC, Gómez Rosso L (2012) HOMA-IR and non-HDL-C as predictors of high cholesteryl ester transfer protein activity in patients at risk for type 2 diabetes. Clin Biochem 45: 566-570. https:// doi.org/10.1016/j.clinbiochem.2012.02.005

Dirimanov S, Högger P (2019) Screening of inhibitory effects of polyphenols on Akt-phosphorylation in endothelial cells and determination of structure-activity features. Biomolecules 9: https://doi. org/10.3390/biom9060219

Dunaif A (1997) Insulin resistance and the polycystic ovary syndrome: mechanism and implications for pathogenesis. Endocr Rev 18: 774 800. https://doi.org/10.1210/edrv.18.6.0318

Escobar-Morreale HF, Luque-Ramírez M, San Millán JLJEr (2005) The molecular-genetic basis of functional hyperandrogenism and the polycystic ovary syndrome. 26: 251-282

Goncharenko VM, Beniuk VA, Kalenska OV, Demchenko OM, Spivak MY, Bubnov RV (2013) Predictive diagnosis of endometrial hyperplasia and personalized therapeutic strategy in women of fertile age. Epma J 4: 24. https://doi.org/10.1186/1878-5085-4-24

Harris RM, Wood DM, Bottomley L, Blagg S, Owen K, Hughes PJ, Kirk CJ (2004) Phytoestrogens are potent inhibitors of estrogen sulfation: implications for breast cancer risk and treatment. J Clin 
Endocrinol Metab 89: 1779-1787. https://doi.org/10.1210/jc.2003031631

Huang S, Okamoto K, Yu C, Sinicrope FA (2013) p62/sequestosome-1 up-regulation promotes ABT-263-induced caspase-8 aggregation/ activation on the autophagosome. J Biol Chem 288: 33654-33666. https://doi.org/10.1074/jbc.M113.518134

Ip PP, Irving JA, McCluggage WG, Clement PB, Young RH (2013) Papillary proliferation of the endometrium: a clinicopathologic study of 59 cases of simple and complex papillae without cytologic atypia. Am J Surg Pathol 37: 167-177. https://doi.org/10.1097/ PAS.0b013e318272d428

Jung CH, Ro SH, Cao J, Otto NM, Kim DH (2010) mTOR regulation of autophagy. FEBS Lett 584: 1287-1295. https://doi. org/10.1016/j.febslet.2010.01.017

Kandemir FM, Yıldırım S, Kucukler S, Caglavan C, Darendelioğlu E, Dortbudak MB (2020) Protective effects of morin against acrylamide-induced hepatotoxicity and nephrotoxicity: A multi-biomarker approach. Food Chem Toxicol 138: 111190. https://doi.org/10.1016/j. fct.2020.111190

Keskin M, Kurtoglu S, Kendirci M, Atabek ME, Yazici C (2005) Homeostasis model assessment is more reliable than the fasting glucose/insulin ratio and quantitative insulin sensitivity check index for assessing insulin resistance among obese children and adolescents. Pediatrics 115: e500-e503. https://doi.org/10.1542/peds.2004-1921

Kuiper GG, Lemmen JG, Carlsson B, Corton JC, Safe SH, van der Saag PT, Gustafsson, JA (1998) Interaction of estrogenic chemicals and phytoestrogens with estrogen receptor beta. Endocrinology 139: 4252-4263. https://doi.org/10.1210/endo.139.10.6216

Kumar V, Verma A, Bhatt PC (2017). 42P - Dual inhibitory effects of novel naringenin analogue in tobacco-carcinogen induced lung cancer via inhibition of PI3K/Akt/mTOR pathway. Ann Oncol 28 : 12-15. https://doi.org/10.1093/annonc/mdx089.013

Kurman RJ, Kaminski PF, Norris HJ (1985) The behavior of endometrial hyperplasia. A long-term study of "untreated" hyperplasia in 170 patients. Cancer 56: 403-412. https://doi.org/10.1002/10970142(19850715) 56

Lee BC, Lee J (2014) Cellular and molecular players in adipose tissue inflammation in the development of obesity-induced insulin resistance. Biochim Biophys Acta 1842: 446-462. https://doi.org/10.1016/j. bbadis.2013.05.017

Lee YJ, Kim WI, Kim SY, Cho SW, Nam HS, Lee SH, Cho MK (2019) Flavonoid morin inhibits proliferation and induces apoptosis of melanoma cells by regulating reactive oxygen species, $\mathrm{Sp} 1$ and Mcl-1. Arch Pharm Res 42: 531-542. https://doi.org/10.1007/ s12272-019-01158-5

Li H, Chen Y, Yan LY, Qiao J (2013) Increased expression of P450scc and CYP17 in development of endogenous hyperandrogenism in a rat model of PCOS. Endocrine 43: 184-190. https://doi. org/10.1007/s12020-012-9739-3

Lin Y, Tan D, Kan Q, Xiao Z, Jiang Z (2018) The protective effect of naringenin on airway remodeling after mycoplasma pneumoniae infection by inhibiting autophagy-mediated lung inflammation and fibrosis. Mediators Inflamm 18: 8753894. https://doi. org/10.1155/2018/8753894

Liu X, Wang N, Fan S, Zheng X, Yang Y, Zhu Y, Zheng J (2016) The citrus flavonoid naringenin confers protection in a murine endotoxaemia model through AMPK-ATF3-dependent negative regulation of the TLR4 signalling pathway. Sci Rep 6: 39735. https://doi. org/10.1038/srep39735

Martínez-Rodríguez OP, Thompson-Bonilla MDR, Jaramillo-Flores ME (2020). Association between obesity and breast cancer: Molecular bases and the effect of flavonoids in signaling pathways. Crit Rev Food Sci Nutr 60: 3770-3792. https://doi.org/10.1080/10408398.20 19.1708262

Medjakovic S, Mueller M, Jungbauer A (2010). Potential health-modulating effects of isoflavones and metabolites via activation of PPAR and AhR. Nutrients 2: 241-279. https://doi.org/10.3390/nu2030241

Miksicek RJ (1994) Interaction of naturally occurring nonsteroidal estrogens with expressed recombinant human estrogen receptor. J Steroid Biochem Mol Biol 49: 153-160. https://doi.org/10.1016/09600760(94)90005-1

Moran LJ, Hutchison SK, Norman RJ, Teede HJ (2011) Lifestyle changes in women with polycystic ovary syndrome. Cochrane Database Syst Rev 2: 134-138 Cd007506. https://doi.org/10.1002/14651858. CD007506.pub2

Neisy A, Zal F, Seghatoleslam A, Alaee S (2019) Amelioration by quercetin of insulin resistance and uterine GLUT4 and ER $\alpha$ gene expression in rats with polycystic ovary syndrome (PCOS). Reprod Fertil Dev 31: 315-323. https://doi.org/10.1071/rd18222

Nicoletti F, Fagone P, Meroni P, McCubrey J, Bendtzen K (2011) mTOR as a multifunctional therapeutic target in HIV infection. Drug Discov Today 16: 715-721. https://doi.org/10.1016/j. drudis.2011.05.008

Nowak E, Sypniewski D, Bednarek I (2020). Morin exerts anti-metastatic, anti-proliferative and anti-adhesive effect in ovarian cancer cells: an in vitro studies. Mol Biol Rep 47: 1965-1978. https://doi. org/10.1007/s11033-020-05293-x

Pal Singh M, Pal Khaket T, Bajpai VK, Alfarraj S, Kim SG, Chen L, Kang SC (2020) Morin hydrate sensitizes hepatoma cells and xenograft tumor towards cisplatin by downregulating PARP-1-HMGB1 mediated autophagy. Int J Mol Sci 21: https://doi.org/10.3390/ ijms 21218253

Palma-Duran SA, Caire-Juvera G, Robles-Burgeño Mdel R, OrtegaVélez MI, Gutiérrez-Coronado Mde L, Almada Mdel C, ChávezSuárez K, Campa-Siqueiros M, Grajeda-Cota P, Saucedo-Tamayo Mdel S, Valenzuela-Quintanar AI (2015) Serum levels of phytoestrogens as biomarkers of intake in Mexican women. Int I Food Sci Nutr 66: 819-825. https://doi.org/10.3109/09637486.2015.1092019

Park JC, Lim SY, Jang TK, Bae JG, Kim JI, Rhee JH (2011) Endometrial histology and predictable clinical factors for endometrial disease in women with polycystic ovary syndrome. Clin Exp Reprod Med 38: 42-46. https://doi.org/10.5653/cerm.2011.38.1.42

Raha S, Yumnam S, Hong GE, Lee HJ, Saralamma VV, Park HS, Kim GS (2015) Naringin induces autophagy-mediated growth inhibition by downregulating the PI3K/Akt/mTOR cascade via activation of MAPK pathways in AGS cancer cells. Int J Oncol 47: 1061-1069. https://doi.org/10.3892/ijo.2015.3095

Rajput SA, Wang Xq, Yan HC (2021) Morin hydrate: A comprehensive review on novel natural dietary bioactive compound with versatile biological and pharmacological potential. Biomed Pharmacother 138: 111511. https://doi.org/10.1016/j.biopha.2021.111511

Rajput SA, Wang XQ, Yan HC (2021) Morin hydrate: A comprehensive review on novel natural dietary bioactive compound with versatile biological and pharmacological potential. Biomed Pharmacother. 138: 111511. https://doi.org/10.1016/j.biopha.2021.111511

Rakha E, Wong SC, Soomro I, Chaudry Z, Sharma A, Deen S, Brown L (2012) Clinical outcome of atypical endometrial hyperplasia diagnosed on an endometrial biopsy: institutional experience and review of literature. Am J Surg Pathol 36: 1683-1690. https://doi. org/10.1097/PAS.0b013e31825dd4ff

Rehman MU, Rahman Mir MU, Faroog A, Rashid SM, Ahmad B, Bilal Ahmad S, Ahmad Ganaie M (2018) Naringenin (4,5,7-trihydroxyflavanone) suppresses the development of precancerous lesions via controlling hyperproliferation and inflammation in the colon of Wistar rats. Environ Toxicol 33: 422-435. https://doi.org/10.1002/ tox. 22528

Roy S, Ahmed F, Banerjee S, Saha U (2016) Naringenin ameliorates streptozotocin-induced diabetic rat renal impairment by downregulation of TGF- $\beta 1$ and IL-1 via modulation of oxidative stress correlates with decreased apoptotic events. Pharm Biol 54: 1616-1627. https: / /doi.org/10.3109/13880209.2015.1110599

Sarkaki A, Farbood Y, Badavi M, Khalaj L, Khodagholi F, Ashabi G (2015) Metformin improves anxiety-like behaviors through AMPKdependent regulation of autophagy following transient forebrain ischemia. Metab Brain Dis 30: 1139-1150. https://doi.org/10.1007/ s11011-015-9677-x

Sarkar A, Angeline MS, Anand K, Ambasta RK, Kumar P (2012) Naringenin and quercetin reverse the effect of hypobaric hypoxia and elicit neuroprotective response in the murine model. Brain Res 1481: 59-70. https://doi.org/10.1016/j.brainres.2012.08.036

Singh MP, Cho HJ, Kim JT, Baek KE, Lee HG, Kang SC (2019) Morin hydrate reverses cisplatin resistance by impairing PARP1/ HMGB1-dependent autophagy in hepatocellular carcinoma. Cancers (Basel) 11: https://doi.org/10.3390/cancers11070986

Taguchi K, Fujikawa N, Komatsu M, Ishii T, Unno M, Akaike T, Yamamoto M (2012) Keap1 degradation by autophagy for the maintenance of redox homeostasis. Proc Natl Acad Sci US A 109: 13561-13566. https://doi.org/10.1073/pnas.1121572109

Tamadon A, Hu W, Cui P, Ma T, Tong X, Zhang F, Feng Y (2018) How to choose the suitable animal model of polycystic ovary syndrome? Trad Med Modern Med 01: 95-113. https://doi.org/10.1142/ s2575900018300047

Tanida I, Ueno T, Kominami E (2008) LC3 and Autophagy. Methods Mol Biol 445: 77-88. https://doi.org/10.1007/978-1-59745-157-4_4

Treeck O, Wackwitz B, Haus U, Ortmann O (2006) Effects of a combined treatment with mTOR inhibitor RAD001 and tamoxifen in vitro on growth and apoptosis of human cancer cells. Gynecol Oncol 102: 292-299. https://doi.org/10.1016/j.ygyno.2005.12.019

Ververidis F, Trantas E, Douglas C, Vollmer G, Kretzschmar G, Panopoulos N (2007) Biotechnology of flavonoids and other phenylpropanoid-derived natural products. Part I: Chemical diversity, impacts on plant biology and human health. Biotechnol J 2: 1214-1234. https://doi.org/10.1002/biot.200700084

Wali AF, Rashid S, Rashid SM, Ansari MA, Khan MR, Haq N, Rehman MU (2020) Naringenin regulates doxorubicin-induced liver dysfunction: impact on oxidative stress and inflammation. Plants (Basel) 9. https://doi.org/10.3390/plants9040550

Xu C, Chen J, Zhang J, Hu X, Jiang HJH (2011) Naringenin inhibits vascular smooth muscle cell function involving reactive oxygen species production modulation and $\mathrm{NF}-x \mathrm{~B}$ activity suppression. Heart 97: A83-A83 
You Q, Wu Z, Wu B, Liu C, Huang R, Yang L, Chen J (2016) Naringin protects cardiomyocytes against hyperglycemia-induced injuries in vitro and in vivo. J Endocrinol 230: 197-214. https://doi. org $/ 10.1530$ /joe-16-0004

Yu J, Zhai D, Hao L, Zhang D, Bai L, Cai Z, Yu C (2014) Cryptotanshinone reverses reproductive and metabolic disturbances in PCOS model rats via regulating the expression of CYP17 and AR. Evid Based Complement Alternat Med 23: 670743. https://doi. org $/ 10.1155 / 2014 / 670743$
Zhang B, Wei YZ, Wang G Q, Li DD, Shi JS, Zhang F (2018) Targeting MAPK pathways by naringenin modulates microglia M1/M2 polarization in lipopolysaccharide-stimulated cultures. Front Cell Neurosci 12: 531. https://doi.org/10.3389/fncel.2018.00531

Zhang Y, Hu M, Meng F, Sun X, Xu H, Zhang J, Billig H (2017) Metformin ameliorates uterine defects in a rat model of polycystic ovary syndrome. EBioMedicine 18: 157-170. https://doi.org/10.1016/j. ebiom.2017.03.023 